\title{
On Rings whose Maximal Ideals are GP-Ideals
}

Raida D. Mahmood

Manal A. Abd

raida.1961@uomosul.edu.iq

College of Computer Sciences and Mathematics

University of Mosul, Iraq

Received on: 28/08/2004

Accepted on: 05/04/2005

\section{ABSTRACT}

This paper introduces the notion of maximal GP-ideal .We studied the class of rings whose maximal left ideal are right GP-ideal. We call such ring MRGP-rings. We consider a necessary and sufficient condition for MRGP-rings to be MRCP-rings. We also study the connection between MRGP-ring, kasch ring, division ring and the strongly regular ring.

Key words: strongaly regular, kasch, GP-ideal, division ring.

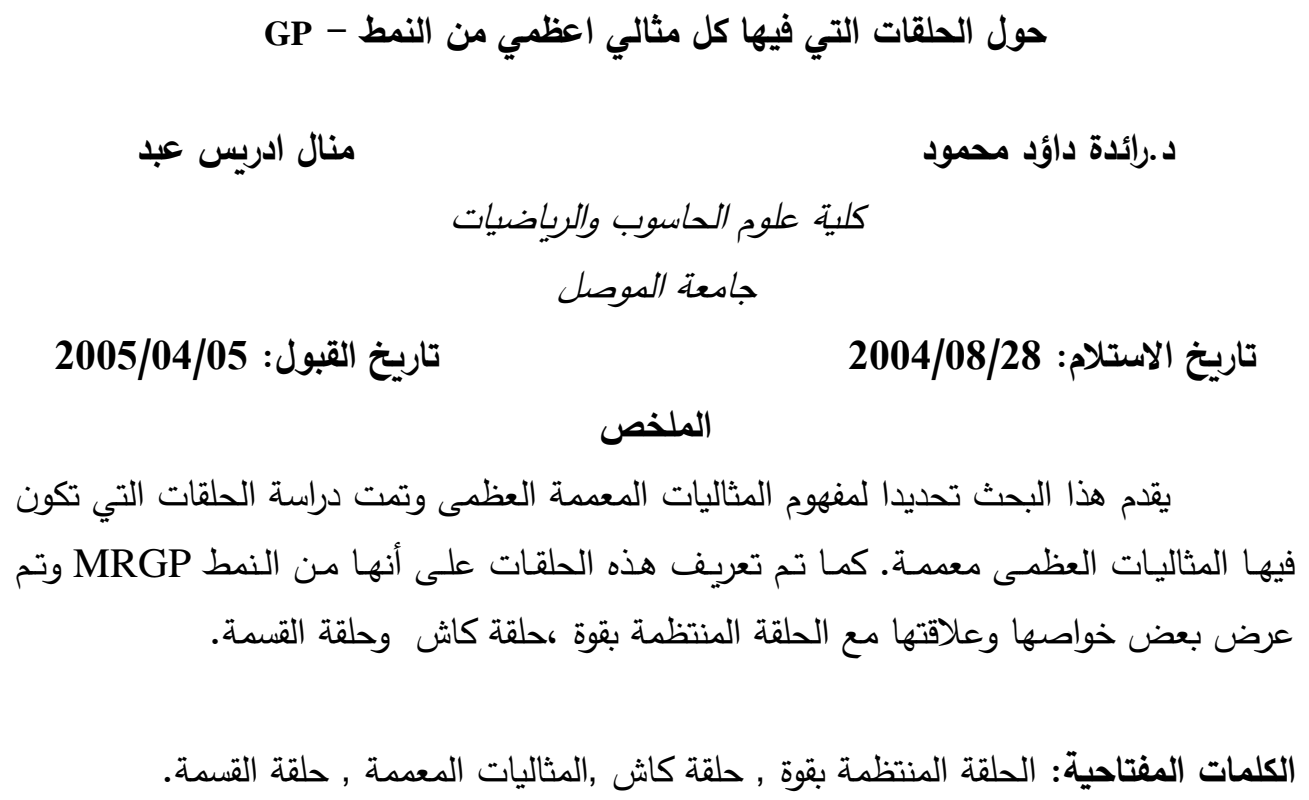




\section{1- Introduction:}

Throughout this paper, $\mathrm{R}$ will denote an associative ring with identity. For any element a in $\mathrm{R}$, we define the right annihilater of a by $r(a)=\{x \in R: a x=0\}$, and likewise the left annihilater $1(a)$. Y,Z,J will denote respectively the right singular ideal, the left singular ideal and the Jacobson radical of R. Recall that 1) An ideal I is said to be a right (left) pure if for every $a \in I$, there exists $b \in I$ such that $a=a b(b a)[1] 2) ~ R$ is called a uniform ring if for every non-zero ideal of $\mathrm{R}$ is essential , [4] .3)

A ring $\mathrm{R}$ is said to be left kasch ring, if every maximal right ideal is a right annihilator [3] 4) $R$ is said to be strongly regular if for each $a \in R$, there exists $x \in R$ such that $a=a^{2} x$. Following [1] . 5) $A$ ring $R$ is called reduced if $R$ has no non-zero nilpotent element and an ideal $I$ of a ring $R$ is said to be right (left) GP-ideals if for every $a \in I$, there exists $b \in I$ and a positive integer $n$ such that $a^{n}=a^{n} b\left(b a^{n}\right)[5]$.

\section{2-MRGP-Rings:}

Following [6] a maximal left ideal $\mathrm{M}$ of the ring $\mathrm{R}$ is said to be a right Co-pure if for every $\mathrm{a} \in \mathrm{M}, \mathrm{Ma}$ is a right pure.

Definition 2-1: A ring $\mathrm{R}$ is called MRCP-ring, if all maximal left ideals are right Co-pure, .see[6]

Definition 2-2: $\mathrm{R}$ is called MRGP-ring, if for any maximal left ideal $\mathrm{M}$ of $\mathrm{R}$, any $\mathrm{a} \in \mathrm{M}, \mathrm{Ma}$ is a right GP-ideal .

Clearly every MRCP-ring is an MRGP-ring, however the converse is not true as the following example shows:

Example: The ring $\mathrm{Z}_{12}$ of integers modulo 12 is an MRGP-ring but not an MRCP-ring.

We now consider a necessary and sufficient condition for MRGPring to be an MRCP-ring.

Theorem 2-3: Let $\mathrm{R}$ be a reduced MRGP-ring. Then $\mathrm{R}$ is MRCP-ring

Proof: Let M be any maximal left ideal of R. Since R is MRGP-ring, then $\mathrm{Ma}$ is a right GP-ideal of $\mathrm{R}$ and there exists $\mathrm{c}, \mathrm{b} \in \mathrm{M}$ and a positive integer $\mathrm{n}$ such that $(\mathrm{ba})^{\mathrm{n}}=(\mathrm{ba})^{\mathrm{n}}(\mathrm{ca})$ this implies that $(\mathrm{ba})^{\mathrm{n}}(1-\mathrm{ca})=0$ and hence (1ca) $\in \mathrm{r}(\mathrm{ba})^{\mathrm{n}} \subseteq \mathrm{r}(\mathrm{ba})$.

Therefore $(1-c a)) \in r(b a)$, whence $(b a)(1-c a)=0$. Thus ba=ba ca (Ma is a right pure) and hence $\mathrm{R}$ is MRCP-ring. 
Lemma 2-4: let a be a non-zero element of the ring $\mathrm{R}$ and let $\mathrm{r}(\mathrm{a})=0$. Then $\mathrm{r}\left(\mathrm{a}^{2 \mathrm{n}}\right)=0$

Theorem 2-5: let $\mathrm{R}$ be an MRGP-ring and $\mathrm{r}(\mathrm{a})=0$. Then $\mathrm{a}$ is a left invertible.

Proof: let $a \in R$ with $r(a)=0$. If $R a \neq R$, there exists a maximal left ideal $M$ containing $\mathrm{Ra}$. Since $\mathrm{R}$ is an MRGP- ring, there exists $\mathrm{b} \in \mathrm{M}$ and a positive integer $n$ such that $a^{2 n}=a^{2 n}(b a)$. Whence $(1-b a) \in r\left(a^{2 n}\right)=0$, yielding $1 \in M$, which contradicts $M \neq R$. Therefore $R a=R$. In particular $r a=1$ for some $r \in R$. Hence $\mathrm{a}$ is a left invertible.

Proposition 2-6: Let $\mathrm{R}$ be a right uniform reduced MRGP-ring. Then $\mathrm{R}$ is a division ring.

Proof: If $0 \neq a \in R$ and $R a \neq R$. Let $M$ be a maximal left ideal containing $R a$. Since $\mathrm{R}$ is MRGP-rings , then for every $\mathrm{a} \in \mathrm{M}$, Ma is a right GP-ideal, so $(\mathrm{ca})^{\mathrm{n}}=(\mathrm{ca})^{\mathrm{n}}$ ba , for some $\mathrm{c}, \mathrm{b} \in \mathrm{M}$ and a positive integer $\mathrm{n}$. Since $\mathrm{R}$ is a right uniform then every right ideal is essential ideal.

Consider $1(\mathrm{ba}) \cap \mathrm{R}(\mathrm{ca})^{\mathrm{n}}$, let $\mathrm{x} \in \mathrm{l}(\mathrm{ba}) \cap \mathrm{R}(\mathrm{ca})^{\mathrm{n}}$ implies that $\mathrm{xba}=0$ and $(\mathrm{ca})^{\mathrm{n}}=\mathrm{x}$,so $(\mathrm{ca})^{\mathrm{n}} \mathrm{ba}=0$, then $(\mathrm{ca})^{\mathrm{n}} \mathrm{ba}=(\mathrm{ca})^{\mathrm{n}}=0$. Therefore $\mathrm{l}(\mathrm{ba}) \cap \mathrm{R}(\mathrm{ca})^{\mathrm{n}}=0$ implies $1(b a)=0$, since $R$ is reduced , $r(b a)=0$. By Theorem $(2-5)$, ba is a left invertible, there exists $\mathrm{y} \in \mathrm{R}$ such that $\mathrm{v}(\mathrm{ba})=1$, so $(\mathrm{vb}) \mathrm{a}=1 \in \mathrm{M}$, a contradiction. Therefore $\mathrm{Ra}=\mathrm{R}$. So $\mathrm{R}$ is division ring.

A ring $\mathrm{R}$ is called zero commutative (briefly $\mathrm{ZC}$ ) [2] if for $\mathrm{a}, \mathrm{b} \in \mathrm{R}$ $\mathrm{ab}=0$ implies $\mathrm{ba}=0$

Proposition(2-7): Let $\mathrm{R}$ be a zero commutative , MRGP-ring. Then $\mathrm{R}$ is a kasch ring .

Proof: Let $\mathrm{M}$ be any maximal left ideal of $\mathrm{R}$, and let $\mathrm{Z}$ be the left singular ideal of $R$, if $M \cap Z=0$, then for any $y \in Z, y \notin M$, this implies that $l(y)$ is an essential left ideal of $\mathrm{R}$.

Let $\mathrm{x} \in \mathrm{l}(\mathrm{y}) \cap 1(1-\mathrm{y})$, then $\mathrm{xy}=0$ and $\mathrm{x}(1-\mathrm{y})=0$, yields $\mathrm{x}=\mathrm{xy}=0$. Therefore $1(\mathrm{y})$ $\bigcap 1(1-y)=0$, whence $1(1-y)=0$.

Since $\mathrm{R}$ is a zero commutative, then we have $\mathrm{r}(1-\mathrm{y})=0$. By Theorem (2-5) $1-y$ is an invertible element of $R$, hence $y \in J \subset M$ a contradiction. Thus $\mathrm{M} \cap \mathrm{Z} \neq 0$.

Let $\mathrm{a} \in \mathrm{M} \cap \mathrm{Z}$, since $\mathrm{R}$ is an MRGP-ring, then Ma is a right GPideal of $\mathrm{R}$ and there exists $\mathrm{c}, \mathrm{b} \in \mathrm{M}$ and a positive integer $\mathrm{n}$ such that 
$(\mathrm{ba})^{\mathrm{n}}=(\mathrm{ba})^{\mathrm{n}}(\mathrm{ca})$. We claim that $\mathrm{l}(\mathrm{ca}) \cap \mathrm{R}(\mathrm{ba})^{\mathrm{n}}=0$, if not let $\mathrm{d} \in \mathrm{l}(\mathrm{ca}) \cap \mathrm{R}(\mathrm{ba})^{\mathrm{n}}$, then $\mathrm{d} c \mathrm{c}=0$ and $\mathrm{d}=\mathrm{r}(\mathrm{ba})^{\mathrm{n}}$ for some $\mathrm{r}$ in $\mathrm{R}$, so $\mathrm{r}(\mathrm{ba})^{\mathrm{n}} \mathrm{ca}=0$, implies $\mathrm{r}(\mathrm{ba})^{\mathrm{n}}=0$, whence $d=0$. Therefore $1(c a) \cap R(b a)^{n}=0$, but $r(c a)$ is essential then $R(b a)^{n}=0$ and hence $b^{n} a^{n}=0$ implies that $b^{n} \in l\left(a^{n}\right)$ and $a^{n} \in r\left(b^{n}\right)$.Therefore $\mathrm{M}=1\left(\mathrm{a}^{\mathrm{n}}\right)$ and $\mathrm{M}=\mathrm{r}\left(\mathrm{b}^{\mathrm{n}}\right)$.

The following theorem gives the condition of being MRGP-ring is strongly regular.

Theorem 2-8: Let $\mathrm{R}$ be a reduced MRGP-ring. Then $\mathrm{R}$ is strongly regular.

Proof: Let $\mathrm{z}$ be a non-zero element in $\mathrm{R}$. We claim that $\mathrm{Rz}+\mathrm{l}(\mathrm{z})=\mathrm{R}$. If $\mathrm{Rz}+\mathrm{l}(\mathrm{z}) \neq \mathrm{R}$, let $\mathrm{M}$ be a maximal left ideal containing $\mathrm{Rz}+\mathrm{l}(\mathrm{z})$. Since $\mathrm{R}$ is an MRGP- ring, then $\mathrm{Mz}$ is a right GP-ideal and there exists $c \in M$ and a positive integer $n$ such that $(z)^{2 n}=(z)^{2 n}(c z)$. Whence $(1-c z) \in r(z)^{2 n}$. Since $R$ is reduced, we have $(1-\mathrm{cz})) \in \mathrm{r}(\mathrm{z})^{2 \mathrm{n}}=\mathrm{l}\left(\mathrm{z}^{2 \mathrm{n}}\right)=\mathrm{l}(\mathrm{z}) \subseteq \mathrm{M}$ this implies that $1 \in \mathrm{M}$, a contradiction .Therefore $\mathrm{Rz}+\mathrm{l}(\mathrm{z})=\mathrm{R}$, in particular $\mathrm{xz}+\mathrm{y}=1, \mathrm{x} \in \mathrm{R}, \mathrm{y} \in \mathrm{l}(\mathrm{z})$.

Thus $\mathrm{z}=\mathrm{xz}^{2}$, and therefore $\mathrm{R}$ is strongly regular. 


\section{FERENRECES}

[1] Al-Ezeh H. (1989), "Pure Ideals in Commutative Reduced Gelfand Ring with unity", Arch. Vol.53.266-269.

[2] Nam S.B, kim N.K. and Kim. J.Y. (1999), "On Simple Singular GPinjective Modules", Comm. In Algebrn 27,(5).

[3] Mohammand M.A.(2003) , Kasch Rings , M.Sc Thesis. Mosul Univ.

[4] Ming. Y.C. (1983), "Maximal ideals in Regular Rings", HokkMath. J. 12,119-128.

[5] Shuker N.H. and Raida D.M. (2000), “On Generalization of Pure Ideals ", J. Edu. And Sci. Vol (43), 86-89.

[6] Shuker N.H. and Raida D.M. (2000), "On Rings Whose Maximal Ideals are Co-Pure”, J. of Science Raf. Vol. 11.,No.(4). 